\title{
$S U(n)$-CONNECTIONS AND
}

\section{NONCOMMUTATIVE DIFFERENTIAL GEOMETRY}

\author{
Michel DUBOIS-VIOLETTE and Thierry MASSON \\ Laboratoire de Physique Théorique et Hautes Energies ${ }^{1}$ \\ Université Paris XI, Bâtiment 211 \\ 91405 Orsay Cedex, France \\ e-mail: flad@qcd.th.u-psud.fr and masson@qcd.th.u-psud.fr
}

\begin{abstract}
We study the noncommutative differential geometry of the algebra of endomorphisms of any $S U(n)$-vector bundle. We show that ordinary connections on such $S U(n)$-vector bundle can be interpreted in a natural way as a noncommutative 1-form on this algebra for the differential calculus based on derivations. We interpret the Lie algebra of derivations of the algebra of endomorphisms as a Lie algebroid. Then we look at noncommutative connections as generalizations of these usual connections.
\end{abstract}

L.P.T.H.E.-ORSAY 96/100

${ }^{1}$ Laboratoire associé au Centre National de la Recherche Scientifique - URA D0063 
The noncommutative differential geometry of the algebra of matrix valued functions on a manifold has been studied in [6]. There it was pointed out that noncommutative connections are good candidate to unify at the classical level ordinary gauge fields and Higgs fields in a unique object. This idea has been widely used in a variety of contexts. In this paper, as a generalization of [6], we consider the noncommutative differential calculus based on derivations for the algebra of endomorphisms of a $S U(n)$-vector bundle.

In Section 1, we recall the definition of the differential calculus based on derivation and the main results for the algebra of matrix valued functions. In Section 2, we consider the algebra of endomorphisms of a $S U(n)$-vector bundle. We show that the affine space of $S U(n)$-connections is an affine subspace of the space of noncommutative 1-form of this algebra. In Section 3, the Lie algebra of derivations of this algebra is interpreted as a Lie algebroid. In Section 4, noncommutative connections are considered. It is shown that they incorporate naturally Higgs fields as in the case of matrix valued functions.

\section{Derivations based noncommutative differen- tial calculi}

The differential calculus based on derivations is a natural generalization the differential algebra of differential forms on a manifold ([3, 4, 7] and references therein).

\subsection{Construction}

Let $\mathfrak{A}$ be an associative algebra with unit. Then one has the well known results:

Lemma 1 The vector space $\operatorname{Der}(\mathfrak{A})$ of derivations of $\mathfrak{A}$ is a Lie algebra and a module over the center $\mathcal{Z}(\mathfrak{A})$ of $\mathfrak{A}$. The center $\mathcal{Z}(\mathfrak{A})$ is stable by $\operatorname{Der}(\mathfrak{A})$. The vector space of inner derivations $\operatorname{Int}(\mathfrak{A})$ is a Lie ideal and a $\mathcal{Z}(\mathfrak{A})$-submodule.

The quotient $\operatorname{Der}(\mathfrak{A}) / \operatorname{Int}(\mathfrak{A})$ will be denoted by $\operatorname{Out}(\mathfrak{A})$. This is then a Lie algebra and a module over $\mathcal{Z}(\mathfrak{A})$.

One can consider the complex $\underline{\Omega}_{\text {Der }}(\mathfrak{A})$ of $\mathcal{Z}(\mathfrak{A})$-multilinear antisymmetric maps from $\operatorname{Der}(\mathfrak{A})$ to $\mathfrak{A}$. It is naturally a $\mathbb{N}$-graded algebra. One defines a 
differential $\hat{d}$ (of degree 1) on this graded algebra by setting, for any derivations $X_{1}, \ldots, X_{n+1}$ and any $\omega \in \underline{\Omega}_{\text {Der }}^{n}(\mathfrak{A})$

$$
\begin{aligned}
& \hat{d} \omega\left(X_{1}, \ldots, X_{n+1}\right)=\sum_{i=1}^{n+1}(-1)^{i+1} X_{i} \omega\left(X_{1}, \ldots \stackrel{i}{\vee} \ldots, X_{n+1}\right) \\
& +\sum_{1 \leq i<j \leq n+1}(-1)^{i+j} \omega\left(\left[X_{i}, X_{j}\right], \ldots \stackrel{i}{\vee} \ldots \stackrel{j}{\vee} \ldots, X_{n+1}\right)
\end{aligned}
$$

The graded differential algebra $\left(\underline{\Omega}_{\text {Der }}(\mathfrak{A}), \hat{d}\right)$ is the first noncommutative differential calculus we define on $\mathfrak{A}$.

The second one is the smallest differential subalgebra of $\underline{\Omega}_{\text {Der }}(\mathfrak{A})$ generated by $\mathfrak{A}$. We denote it by $\Omega_{\text {Der }}(\mathfrak{A})$. Any element of $\Omega_{\text {Der }}^{n}(\mathfrak{A})$ is a sum of terms $a_{0} \hat{d} a_{1} \ldots \hat{d} a_{n}$ for $a_{i} \in \mathfrak{A}$.

These definitions are generalizations of the usual differential calculus on a manifold in the sense that when $\mathfrak{A}$ is the algebra $C^{\infty}(M)$ of smooth functions on a finite dimensional regular smooth manifold $M$, these differential algebras coincide with the graded differential algebra of differential forms on $M$.

\subsection{The matrix algebra}

The noncommutative geometry of the matrix algebra for the differential calculus based on derivations has been described in [4] and [5]. We briefly summarize here the results.

The algebra $M_{n}(\mathbb{C})$ has only inner derivations. The Lie algebra $\operatorname{Der}\left(M_{n}(\mathbb{C})\right)=$ $\operatorname{Int}\left(M_{n}(\mathbb{C})\right)$ can be identified with the Lie algebra $\operatorname{sl}(n, \mathbb{C})$. The two previously defined differential calculi are the same and one has

$$
\Omega_{\text {Der }}\left(M_{n}(\mathbb{C})\right)=M_{n}(\mathbb{C}) \otimes \bigwedge \operatorname{sl}(n, \mathbb{C})^{*}
$$

where $\operatorname{sl}(n, \mathbb{C})^{*}$ is the dual of $\operatorname{sl}(n, \mathbb{C})$. We denote by $d^{\prime}$ the differential on this complex.

There exists a particular 1-form $\theta$ defined by

$$
i \theta\left(a d_{\gamma}\right)=\gamma-\frac{1}{n} \operatorname{Tr}(\gamma) \mathbb{1}
$$


for any $\gamma \in M_{n}(\mathbb{C})$. This 1 -form satisfies

$$
d^{\prime} i \theta-(i \theta)^{2}=0
$$

and for any $\gamma \in M_{n}(\mathbb{C})=\Omega_{\operatorname{Der}}^{0}\left(M_{n}(\mathbb{C})\right)$, one has $d^{\prime} \gamma=[i \theta, \gamma]$.

\subsection{The matrix valued functions algebra}

The derivations based differential calculus for the algebra $\mathfrak{A}=C^{\infty}(M) \otimes M_{n}(\mathbb{C})$ for a manifold $M$ has been studied in [6]. The main results are the following.

The center of the algebra $\mathfrak{A}$ is the algebra $C^{\infty}(M)$ of smooth complex valued functions on $M$. The Lie algebra of derivations $\operatorname{Der}(\mathfrak{A})$ split canonically as a $C^{\infty}(M)$-module into

$$
\operatorname{Der}(\mathfrak{A})=\left[\operatorname{Der}\left(C^{\infty}(M)\right) \otimes \mathbb{1}\right] \oplus\left[C^{\infty}(M) \otimes \operatorname{Der}\left(M_{n}(\mathbb{C})\right)\right]
$$

where $\operatorname{Der}\left(C^{\infty}(M)\right)=\Gamma(T M)$ is the ordinary Lie algebra of vector fields on $M$ and $\operatorname{Der}\left(M_{n}(\mathbb{C})\right)=\operatorname{sl}(n, \mathbb{C})$.

This result implies the canonical decomposition

$$
\Omega_{\text {Der }}(\mathfrak{A})=\Omega(M) \otimes \Omega_{\text {Der }}\left(M_{n}(\mathbb{C})\right)
$$

where $\Omega(M)$ is the graded differential algebra of differential forms on $M$, with differential $d$. The differential $\hat{d}$ on $\Omega_{\text {Der }}(\mathfrak{A})$ is the sum $\hat{d}=d+d^{\prime}$. In particular, restricted to $\mathfrak{A}, \hat{d}=d+a d_{i \theta}$.

The 1 -form $\theta$ is well defined in $\Omega_{\text {Der }}^{1}(\mathfrak{A})$ if we extend it on $\operatorname{Der}(\mathfrak{A})$ by zero on the $\Gamma(T M)$ terms. $\theta$ is real in the sense that it is real-valued on real (i.e. hermitian) derivations.

\section{The algebra of endomorphisms of a vector bundle}

In the following, by a $S U(n)$-vector bundle $E$ we mean a hermitian vector bundle of rank $n$ such that $\bigwedge^{n} E$ is trivial (i.e. trivializable with a given trivialization).

Let $E$ be a $S U(n)$-vector bundle over regular finite dimensional smooth (i.e. paracompact, etc...) manifold $M$, and let us denote by $\operatorname{End}(E)$ the fiber bundle 
of endomorphisms of $E$. The sections of this fiber bundle in matrix algebras form a unital algebra, which we denote by $\mathfrak{A}$. The hermitian structure gives a natural involution on this algebra, which we denote by $S \mapsto S^{*}$. The center of this algebra is exactly $C^{\infty}(M)$ (smooth functions on $M$ with values in $\mathbb{C}$ ), identifying $f \in C^{\infty}(M)$ with $f \mathbb{1} \in \mathfrak{A}$. The trace map, defined on each fiber of $\operatorname{End}(E)$, gives a natural map

$$
\operatorname{Tr}: \mathfrak{A} \rightarrow C^{\infty}(M)
$$

Similarly, the determinant defines a natural map

$$
\operatorname{det}: \mathfrak{A} \rightarrow C^{\infty}(M)
$$

By restriction to the center, there is a natural map

$$
\rho: \operatorname{Der}(\mathfrak{A}) \rightarrow \operatorname{Der}\left(C^{\infty}(M)\right)=\Gamma(T M)
$$

This map is the quotient map in the short exact sequence of Lie algebras and $C^{\infty}(M)$-modules

$$
0 \rightarrow \operatorname{Int}(\mathfrak{A}) \rightarrow \operatorname{Der}(\mathfrak{A}) \stackrel{\rho}{\longrightarrow} \operatorname{Out}(\mathfrak{A})=\Gamma(T M) \rightarrow 0
$$

For any derivation $\mathcal{X} \in \operatorname{Der}(\mathfrak{A})$, let us denote by $X \in \Gamma(T M)$ the associated vector field on $M$. Notice now that the 1 -form $i \theta$ defined in the previous section is well defined here on $\operatorname{Int}(\mathfrak{A})$ :

$$
i \theta\left(a d_{\gamma}\right)=\gamma-\frac{1}{n} \operatorname{Tr}(\gamma) \mathbb{1}
$$

for any $\gamma \in \mathfrak{A}$. Any inner derivative $a d_{\gamma}$ will be taken such that $\gamma$ is traceless. It is then a section of the fiber bundle of traceless endomorphisms of $E$. We denote by $\mathfrak{A}_{0}$ the set of such sections. The Lie algebra $\operatorname{Int}(\mathfrak{A}) \simeq \mathfrak{A}_{0}$ operates in the sense of $H$. Cartan on $\Omega_{\text {Der }}(\mathfrak{A})[1,3]$. The horizontal elements for this operation can be considered as differential forms on $M$ with values in $\operatorname{End}(E)$. As will be seen below, the curvature of a connection on $E$ can be interpreted in this way. The basic forms are ordinary differential forms on $M$. In the following, horizontality will refer to this operation. 
The subalgebra $\mathcal{Z}(\mathfrak{A})$ of $\mathfrak{A}$ can be considered as a quotient manifold algebra in the sense of [9]. In this interpretation, the algebra $\mathfrak{A}$ looks like a principal bundle and $\mathcal{Z}(\mathfrak{A})$ as the algebra of functions on the base space. The Lie algebra of the gauge group, defined as the Lie algebra of derivations which are zero on $\mathcal{Z}(\mathfrak{A})$, is then $\operatorname{Int}(\mathfrak{A})$. As we will see later, this point of view will be confirmed when we will consider ordinary connections on $E$.

A derivation $\mathcal{X} \in \operatorname{Der}(\mathfrak{A})$ will be said real if $(\mathcal{X} S)^{*}=\mathcal{X}\left(S^{*}\right)$ for any $S \in \mathfrak{A}$. By duality, one defines hermitian and antihermitian noncommutative differential forms.

Let us now look at the differential calculi based on derivations.

Proposition 2 The two differential calculi $\Omega_{\text {Der }}(\mathfrak{A})$ and $\underline{\Omega}_{\text {Der }}(\mathfrak{A})$ coincide.

Indeed, by using a finite open covering of $M$ with open sets which trivialize $E$, and results on smooth matrix valued functions, one can show by standard arguments that any element of $\underline{\Omega}_{\text {Der }}^{p}(\mathfrak{A})$ can be written as a finite sum of terms $a_{0} \hat{d} a_{1} \ldots \hat{d} a_{p}$ with $a_{i} \in \mathfrak{A}$. It is then an element of $\Omega_{\text {Der }}^{p}(\mathfrak{A})$.

We will denote by $\hat{d}$ the differential on $\Omega_{\text {Der }}(\mathfrak{A})=\underline{\Omega_{\text {Der }}}(\mathfrak{A})$.

In the trivial case $\left(E=M \times \mathbb{C}^{n}\right)$, the algebra $\mathfrak{A}$ is exactly $C^{\infty}(M) \otimes M_{n}(\mathbb{C})$, and we are back to the situation of the previous section. In particular, the short exact sequence (1) splits canonically as $C^{\infty}(M)$-modules.

In the general case, one has the following situation.

Proposition 3 Let $\nabla^{E}$ be any connection on $E$. Then there exists a noncommutative 1 -form $\alpha$ in $\Omega_{\text {Der }}^{1}(\mathfrak{A})$ such that any derivation $\mathcal{X} \in \operatorname{Der}(\mathfrak{A})$ can be decomposed as

$$
\mathcal{X}=\nabla_{X}-a d_{\alpha(\mathcal{X})}
$$

where $\nabla$ is the naturally associated connection to $\nabla^{E}$ on the fiber bundle $\operatorname{End}(E)$. In other words, restricted to $\mathfrak{A}$, the noncommutative differential $\hat{d}$ can be written with obvious notation

$$
\hat{d}=\nabla-a d_{\alpha}
$$


We recall that $\nabla$ is the tensor product of the connections $\nabla^{E}$ on $E$ and $\nabla^{E^{*}}$ on the dual vector bundle $E^{*}$ of $E$ where $\nabla^{E^{*}}$ satisfies $X\langle\epsilon, e\rangle=\left\langle\nabla_{X}^{E^{*}} \epsilon, e\right\rangle+\left\langle\epsilon, \nabla_{X}^{E} e\right\rangle$ for any sections $\epsilon$ of $E^{*}$ and $e$ of $E$.

Proof: First, notice that for a fixed $X \in \Gamma(T M), \nabla_{X}: \mathfrak{A} \rightarrow \mathfrak{A}$ is naturally a derivation. Now, the image in $\Gamma(T M)$ of the derivation $\mathcal{X}-\nabla_{X}$ is zero, as can be seen by applying it on any function in $C^{\infty}(M)$. So the derivation $\mathcal{X}-\nabla_{X}$ has to be in the kernel of the quotient map. It is then inner. Define $\alpha$ by

$$
\alpha(\mathcal{X})=-i \theta\left(\mathcal{X}-\nabla_{X}\right)
$$

This is obviously a noncommutative 1 -form on $\mathfrak{A}$ which satisfies by definition $a d_{\alpha(\mathcal{X})}=\nabla_{X}-\mathcal{X}$

Notice that this noncommutative 1-form takes its values in the traceless elements of $\mathfrak{A}$. In fact, one can see $\alpha$ as an extension of $-i \theta$ to all derivations. Indeed, one has obviously $\alpha\left(a d_{\gamma}\right)=-\gamma$. Recall the convention that $\operatorname{Tr}(\gamma)=0$.

This proposition gives us a splitting of the short exact sequence (1) of $C^{\infty}(M)$ modules. This splitting is not canonical and is only defined through a choice of a connection on $E$, by the $C^{\infty}(M)$-linear map $X \mapsto \nabla_{X}$ from $\Gamma(T M)$ into $\operatorname{Der}(\mathfrak{A})$. This has to be compared with the usual (commutative) situation where one can interpret a connection as a map from vector fields on $M$ into vector fields on a principal bundle over $M$. In our situation, the algebra $\mathfrak{A}$ plays a similar role to this principal bundle.

Let us now look at this noncommutative 1-form $\alpha$ in local expressions. On any open subset $U \subset M$ of a trivialization of $\operatorname{End}(E)$, the algebra $\mathfrak{A}$ looks like $C^{\infty}(U) \otimes M_{n}(\mathbb{C})$. Let $S \in \mathfrak{A}$ be a section. Restricted to $U$, this section can be regarded as a matrix valued function $s: U \rightarrow M_{n}(\mathbb{C})$. Let $U^{\prime} \subset M$ be a second open subset of a trivialization of $\operatorname{End}(E)$. The restriction to $U^{\prime}$ of the section $S$ is an other matrix valued function $s^{\prime}: U^{\prime} \rightarrow M_{n}(\mathbb{C})$ which is related to $s$ by $s^{\prime}=g^{-1} s g$ for a transition function $g: U \cap U^{\prime} \rightarrow S U(n)$.

A derivation $\mathcal{X}$ of the algebra $\mathfrak{A}$ can also be trivialized. Because the algebra looks locally as $C^{\infty}(U) \otimes M_{n}(\mathbb{C})$, this trivialization has the form $\mathcal{X}_{\mathrm{loc}}=X+a d_{\gamma}$ 
for $X$ an ordinary vector fields on $U$ and $\gamma$ a traceless matrix valued function on $U$. $X$ is nothing but the restriction to $U$ of the image of $\mathcal{X}$ in $\Gamma(T M)$. On $U^{\prime}$ as above, the trivialization of $\mathcal{X}$ has the form $\mathcal{X}_{\text {loc }}^{\prime}=X+a d_{\gamma^{\prime}}$. It is easy to show that on $U \cap U^{\prime}$, one then has

$$
\gamma^{\prime}=g^{-1} \gamma g+g^{-1}(X g)
$$

Because the structure group is $S U(n)$, the last term is traceless.

Corollary 4 The local expression of the noncommutative 1-form $\alpha$ is

$$
\alpha_{\mathrm{loc}}\left(\mathcal{X}_{\mathrm{loc}}\right)=A(X)-\gamma
$$

where $A$ is the local expression of the connection 1-form of $\nabla^{E}$ (with values in the Lie algebra $s u(n))$.

One can verify directly that those local expressions can be joined together into the global objet $\alpha$ because the inhomogeneous term of the transition relations of the $A$ fields cancels exactly the inhomogeneous term of the transition relations of the $\gamma$ fields. Notice the importance to work on a $S U(n)$-vector bundle. The traceless of the connection 1-form is essential here. Strictly speaking, $S L(n, \mathbb{C})$ connections would be sufficient.

Corollary 5 The canonical mapping $\nabla^{E} \mapsto \alpha$ is an isomorphism of affine spaces from the affine space of $S U(n)$-connections on $E$ onto the affine space of traceless antihermitian noncommutative 1-forms on $\mathfrak{A}$ satisfying $\alpha\left(a d_{\gamma}\right)=-\gamma$.

Indeed, a noncommutative 1-form $\alpha$ is the image of a $S U(n)$-connection if and only if $\alpha\left(a d_{\gamma}\right)=-\gamma$ for any $\gamma \in \mathfrak{A}_{0}, \operatorname{Tr} \alpha(\mathcal{X})=0$, and $\alpha(\mathcal{X})^{*}+\alpha(\mathcal{X})=0$ for any real $\mathcal{X} \in \operatorname{Der}(\mathfrak{A})$. As we will see below, this last condition can be interpreted as a compatibility condition with a hermitian form. Now, one can work on the noncommutative 1-form $\alpha$ instead of $\nabla^{E}$.

Proposition 6 The Lie algebra of real derivations on $\mathfrak{A}$ acts on the space of $S U(n)$-connections through the Lie derivative defined on $\Omega_{\operatorname{Der}}(\mathfrak{A})$. 
Restricted to inner real derivations, the Lie derivative corresponds to infinitesimal gauge transformations on connections.

Proof: It is easy to see that for any real derivation $\mathcal{X} \in \operatorname{Der}(\mathfrak{A})$, if $\alpha$ is the image of a $S U(n)$-connection on $E$, then $\mathcal{L}_{\mathcal{X}} \alpha=\left(i_{\mathcal{X}} \hat{d}+\hat{d} i_{\mathcal{X}}\right) \alpha$ satisfies

$$
\left(\mathcal{L}_{\mathcal{X}} \alpha\right)\left(a d_{\gamma}\right)=0
$$

and

$$
\left(\mathcal{L}_{\mathcal{X}} \alpha\right)(\mathcal{Y})^{*}+\left(\mathcal{L}_{\mathcal{X}} \alpha\right)(\mathcal{Y})=0
$$

for any $\gamma \in \mathfrak{A}_{0}$ and real $\mathcal{Y} \in \operatorname{Der}(\mathfrak{A})$. Then $\alpha+\mathcal{L}_{\mathcal{X}} \alpha$ is the image of a $S U(n)$ connection on $E$. Now, for $\mathcal{X}=a d_{\xi}$ with $\xi^{*}+\xi=0$, one has

$$
\mathcal{L}_{a d_{\xi}} \alpha=-\hat{d} \xi-[\alpha, \xi]
$$

This is exactly an infinitesimal gauge transformation on $\nabla^{E}$ if $\xi$ is considered as an element of the Lie algebra of the group of gauge transformations of $E$.

Notice that in this proof, we have used the fact that the real inner derivations $a d_{\xi}$ are exactly in correspondence with the elements $\xi$ of the Lie algebra of the gauge group of $E$.

In ordinary differential geometry, the connection $\nabla^{E}$ on $E$ is related to a covariant vector valued 1-form on an associated principal bundle (the bundle of $S U(n)$-frames of $E$ ). Here, as we have seen above, the algebra $\mathfrak{A}$ replaces in a certain sense this principal bundle. The connection $\nabla^{E}$ is then associated to a noncommutative 1-form on $\mathfrak{A}$. However, one has to notice two important facts. First, there is no need to a covariance restriction. Instead, one fixes the value of the 1-form on inner derivations. Second, there is a natural way to generalize this structure taking any noncommutative 1-form on $\mathfrak{A}$. In Section 4 we shall see that any noncommutative 1-form represents a noncommutative connection and gives rise to a Yang-Mills-Higgs type multiplet.

Proposition 7 Let $\alpha$ be the noncommutative 1-form associated to the connection $\nabla^{E}$ on $E$. Let us denote by $R^{E}$ the curvature of $\nabla^{E}$. Then one has

$$
R^{E}(X, Y)=\hat{d} \alpha(\mathcal{X}, \mathcal{Y})+[\alpha(\mathcal{X}), \alpha(\mathcal{Y})]
$$


for any $\mathcal{X}, \mathcal{Y} \in \operatorname{Der}(\mathfrak{A}), X, Y$ being their images in $\Gamma(T M)$. In particular, the expression $\hat{d} \alpha+\alpha^{2}$ is a horizontal element of $\Omega_{\text {Der }}^{2}(\mathfrak{A})$.

Proof: The direct computation of $\hat{d} \alpha(\mathcal{X}, \mathcal{Y})+[\alpha(\mathcal{X}), \alpha(\mathcal{Y})]$ in a local trivialization gives immediately $d A(X, Y)+[A(X), A(Y)]$.

This proposition gives us an interpretation of the curvature $R^{E}$ in terms of a horizontal noncommutative 2 -form on $\mathfrak{A}$.

The curvature of the associated connection $\nabla$ on $\operatorname{End}(E)$ is $R=a d_{R^{E}}$. One verifies that the decomposition $\nabla_{X}=\mathcal{X}+a d_{\alpha(\mathcal{X})}$ yields directly to

$$
R(X, Y)=a d_{\hat{d} \alpha(\mathcal{X}, \mathcal{Y})+[\alpha(\mathcal{X}), \alpha(\mathcal{Y})]}
$$

The curvature of $\nabla^{E}$ can be interpreted as the obstruction on the morphism of modules from vector fields on $M$ into vector fields on the associated principal bundle, to be a morphism of Lie algebras. The above formula can be interpreted in a similar way: $R$ is the obstruction on the application $X \mapsto \nabla_{X}$ to be a morphism of Lie algebras from $\Gamma(T M)$ to $\operatorname{Der}(\mathfrak{A})$.

\section{$3 \operatorname{Der}(\mathfrak{A})$ as a Lie algebroid}

There is a deep connection between the above discussion and the notion of Lie algebroid. Let us recall first the definition of Lie algebroids [8].

A Lie algebroid is a vector bundle $L$ over a smooth manifold $M$ with a structure of Lie algebra on its smooth sections and a vector bundle morphism $\rho: L \rightarrow T M$, called the anchor, such that

$$
[\rho(a), \rho(b)]=\rho([a, b])
$$

and

$$
[a, f b]=f[a, b]+(\rho(a) f) b
$$

where $a$ and $b$ are sections of $L,[$,$] is the Lie bracket on sections of L$ (or on vector fields on $M$ in the first formulae) and $f$ is a function on $M$ (the anchor being naturally defined on sections of $L$ ). 
One important example of Lie algebroid is the Atiyah algebra $\mathcal{A}(E)$ associated to any vector bundle $E$ over $M$. It is defined as the Lie algebra of first-order differential operators on $E$ with symbol $\operatorname{Id}_{E} \otimes X$ for $X$ a vector field on $M$, the anchor being the symbol map $\sigma: \mathcal{A}(E) \rightarrow \Gamma(T M)$. One has a natural short exact sequence

$$
0 \rightarrow \Gamma(\operatorname{End}(E)) \rightarrow \mathcal{A}(E) \stackrel{\sigma}{\longrightarrow} \Gamma(T M) \rightarrow 0
$$

of sections of vector bundles.

Associated to any Lie algebroid $L$ there is a differential calculus on the sections of $\bigwedge L^{*}$ with differential $d_{L}$ given by

$$
\begin{aligned}
d_{L} \omega\left(\ell_{1}, \ldots, \ell_{n+1}\right)= & \sum_{i=1}^{n+1}(-1)^{i+1} \rho\left(\ell_{i}\right) \omega\left(\ell_{1}, \ldots \stackrel{i}{.} \ldots, \ell_{n+1}\right) \\
& +\sum_{1 \leq i<j \leq n+1}(-1)^{i+j} \omega\left(\left[\ell_{i}, \ell_{j}\right], \ldots \stackrel{i}{\mathrm{i}} \ldots \stackrel{j}{\mathrm{v}} \ldots, \ell_{n+1}\right)
\end{aligned}
$$

for any $\omega \in \bigwedge^{n} L^{*}$ and $\ell_{1}, \ldots, \ell_{n+1} \in L$.

With previous notations, a $L$-connection on $E$ is an anchor preserving map

$$
D: L \rightarrow \mathcal{A}(E)
$$

The curvature of such a connection is defined as the obstruction to be a Lie algebras morphism.

Consider now $E$ as in the previous section. Then $\operatorname{Der}(\mathfrak{A})$ is in a natural way the sections of a Lie algebroid, with anchor given by the restriction $\rho$ to $C^{\infty}(M)=\mathcal{Z}(\mathfrak{A})$ of derivations of $\mathfrak{A}$ (see the sequence (1)). It is easy to see that $\underline{\Omega}_{\text {Der }}(\mathfrak{A})$ is just $\mathfrak{A} \otimes_{\mathcal{Z}(\mathfrak{A})} \bigwedge_{\mathcal{Z}(\mathfrak{a})} \operatorname{Der}(\mathfrak{A})^{*}$ and the differential associated to the Lie algebroid $\operatorname{Der}(\mathfrak{A})$ defined on $\bigwedge_{\mathcal{Z}(\mathfrak{a})} \operatorname{Der}(\mathfrak{A})^{*}$ as above $(3)$ is just the restriction of $\hat{d}$.

There is a natural anchor preserving map $\mathcal{A}(E) \rightarrow \operatorname{Der}(\mathfrak{A})$ which associates to any $T \in \mathcal{A}(E)$, the derivation $S \mapsto[T, S]$ for any $S \in \mathfrak{A}$ where the commutator is that of operators on $E$. Locally, it is also given by $\operatorname{Id}_{E} \otimes X+A \mapsto X+a d_{A}$ with obvious notations. This map, together with previously defined ones, gives us the following commutative exact diagram of $C^{\infty}(M)$-modules and Lie algebras: 


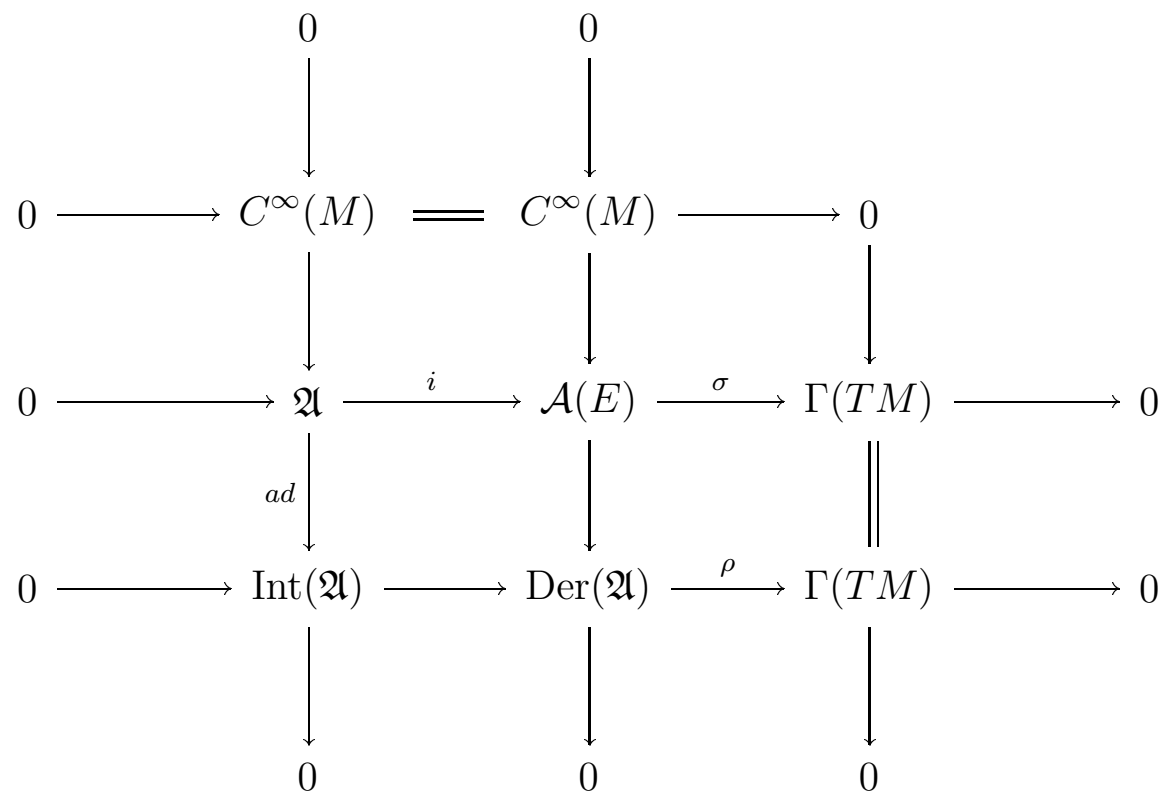

In this diagram, $i \theta: \operatorname{Int}(\mathfrak{A}) \rightarrow \mathfrak{A}$ gives a splitting as $C^{\infty}(M)$-modules and Lie algebras of the first column. Any ordinary connection on $E$ splits the short exact sequence of the second line by $X \mapsto \nabla_{X}^{E}$ and of the third line by $X \mapsto \nabla_{X}$. Any noncommutative 1 -form is a map from $\operatorname{Der}(\mathfrak{A})$ to $\mathfrak{A}$. So, the difference of two $\operatorname{Der}(\mathfrak{A})$-connections in the sense of Lie algebroids is a noncommutative 1-form on $\mathfrak{A}$.

Proposition 8 For any given $S U(n)$-connection $\nabla^{E}$ on $E$ and any $\mathcal{X} \in \operatorname{Der}(\mathfrak{A})$, define $\stackrel{\circ}{D}: \operatorname{Der}(\mathfrak{A}) \rightarrow \mathcal{A}(E)$ by

$$
\stackrel{o}{D}(\mathcal{X})=\nabla_{X}^{E}-\alpha(\mathcal{X})
$$

Then one has the following:

(i) $\stackrel{o}{D}$ is independent of the choice of $\nabla^{E}$,

(ii) $\stackrel{o}{D}$ is a splitting of the short exact sequence

$$
0 \rightarrow C^{\infty}(M) \rightarrow \mathcal{A}(E) \rightarrow \operatorname{Der}(\mathfrak{A}) \rightarrow 0
$$

considered as an exact sequence of $C^{\infty}(M)$-modules as well as an exact sequence of Lie algebras, 
(iii) $\stackrel{\circ}{D}$ induces the splitting $i \theta$ of

$$
0 \rightarrow C^{\infty}(M) \rightarrow \mathfrak{A} \rightarrow \operatorname{Int}(\mathfrak{A}) \rightarrow 0
$$

It follows in particular that $\stackrel{\circ}{D}$ is a $\operatorname{Der}(\mathfrak{A})$-connection with vanishing curvature.

Proof: For any given $S U(n)$-connection $\nabla^{E}$ on $E$, with definition (4), for any $\mathcal{X} \in \operatorname{Der}(\mathfrak{A})$ and any $S \in \mathfrak{A}$, one has $[\stackrel{o}{D}(\mathcal{X}), S]=\nabla_{X} S-[\alpha(\mathcal{X}), S]=\mathcal{X} S$. So $\stackrel{o}{D}$ is a splitting of (5) as $C^{\infty}(M)$-modules. For any $\mathcal{X}, \mathcal{Y} \in \operatorname{Der}(\mathfrak{A})$, one can show by direct computation, using Proposition 7, that $[\stackrel{\circ}{D}(\mathcal{X}), \stackrel{o}{D}(\mathcal{Y})]-\stackrel{o}{D}([\mathcal{X}, \mathcal{Y}])=0$. So $\stackrel{\circ}{D}$ is a splitting of (5) as Lie algebras. Restricted to inner derivations, $\stackrel{\circ}{D}$ coincides with $i \theta$. Then $\stackrel{o}{D}$ induces the splitting of (6).

If $\nabla^{\prime E}$ is an other $S U(n)$-connection on $E$, then $\nabla_{X}^{\prime E}-\alpha^{\prime}(\mathcal{X})-\left(\nabla_{X}^{E}-\alpha(\mathcal{X})\right)=$ $\nabla_{X}^{\prime E}-\nabla_{X}^{E}-i \theta\left(\nabla_{X}^{\prime}-\nabla_{X}\right)$, which is zero because of the relations between $\nabla^{E}$ and $\nabla$.

Is is obvious that $\stackrel{o}{D}$ is anchor preserving, and is then a $\operatorname{Der}(\mathfrak{A})$-connection. Because it is a splitting of (5) as Lie algebras, its curvature vanishes.

Notice that in a local trivialization of $E, \stackrel{o}{D}$ is given by $X+a d_{\gamma} \mapsto \operatorname{Id}_{E} \otimes X+\gamma$ where as usual, $\gamma$ is traceless.

Any other splitting of (5) as $C^{\infty}(M)$-modules and Lie algebras is of the form $\mathcal{X} \mapsto \stackrel{o}{D}(\mathcal{X})+\phi(X)$ with $\phi \in \Omega^{1}(M)$ and $d \phi=0$. In particular, it always induces the splitting $i \theta$. If one drops out the splitting as Lie algebras, then any other splitting of $(5)$ as $C^{\infty}(M)$-modules is of the form $\mathcal{X} \mapsto \stackrel{o}{D}(\mathcal{X})+\phi(\mathcal{X})$ with $\phi$ a noncommutative 1-form on $\mathfrak{A}$ with values in $C^{\infty}(M)$. The curvature of such a splitting is $\hat{d} \phi$. Then $\stackrel{o}{D}$ identifies $\operatorname{Der}(\mathfrak{A})$ as a Lie subalgebroid of $\mathcal{A}(E)$. This Lie subalgebroid is the set of elements of $\mathcal{A}(E)$ which preserve the volume on $E$. In this identification, $\operatorname{Int}(\mathfrak{A})$ is mapped to $\mathfrak{A}_{0}$.

\section{Noncommutative connections}

Let us now turn to noncommutative connections for the differential calculus $\Omega_{\text {Der }}(\mathfrak{A})$ on the previously defined algebra $\mathfrak{A}$. We consider here connections on 
right or left $\mathfrak{A}$-modules. These noncommutative connections have been defined in [2] and used in many approaches to noncommutative geometry.

\subsection{The right module $\mathfrak{A}$}

The simplest example is the right module $\mathfrak{A}$ itself which is the free right $\mathfrak{A}$-module of rank 1. On this right module there is a natural hermitian form defined by $\left\langle S, S^{\prime}\right\rangle=S^{*} S^{\prime} \in \mathfrak{A}$ for any $S, S^{\prime} \in \mathfrak{A}$. In this case, a noncommutative connection is an application

$$
\widehat{\nabla}_{\mathcal{X}}: \mathfrak{A} \rightarrow \mathfrak{A}
$$

such that $\widehat{\nabla}_{\mathcal{X}}\left(S S^{\prime}\right)=S \mathcal{X}\left(S^{\prime}\right)+\widehat{\nabla}_{\mathcal{X}}(S) S^{\prime}$ and $\widehat{\nabla}_{f \mathcal{X}} S=f \widehat{\nabla}_{\mathcal{X}} S$ for any $S, S^{\prime} \in \mathfrak{A}$ and $f \in \mathcal{Z}(\mathfrak{A})$. The curvature of a noncommutative connection is defined by $\hat{R}(\mathcal{X}, \mathcal{Y}) S=\left[\widehat{\nabla}_{\mathcal{X}}, \widehat{\nabla}_{\mathcal{Y}}\right] S-\widehat{\nabla}_{[\mathcal{X}, \mathcal{Y}]} S$ for any $S \in \mathfrak{A}$ and $\mathcal{X}, \mathcal{Y} \in \operatorname{Der}(\mathfrak{A})$, which is a right $\mathfrak{A}$-module homomorphism.

Any noncommutative right-connection $\widehat{\nabla}$ on $\mathfrak{A}$ is entirely given by $\widehat{\nabla}_{\mathcal{X}} \mathbb{1}=$ $\omega(\mathcal{X})$ where $\omega$ is a noncommutative 1 -form in $\Omega_{\text {Der }}^{1}(\mathfrak{A})$. One can then write

$$
\widehat{\nabla}_{\mathcal{X}} S=\mathcal{X} S+\omega(\mathcal{X}) S
$$

for any $S \in \mathfrak{A}$. The expression $\widehat{\nabla}_{\mathcal{X}}^{0} S=\mathcal{X} S$ defines a noncommutative connection on $\mathfrak{A}$ whose curvature is 0 . This is a particular point in the affine space of noncommutative connections on the right module $\mathfrak{A}$. It is easy to see that the curvature of $\widehat{\nabla}$ is the left multiplication by the noncommutative 2 -form

$$
\hat{d} \omega(\mathcal{X}, \mathcal{Y})+[\omega(\mathcal{X}), \omega(\mathcal{Y})]
$$

A connection is said to be compatible with the hermitian structure if

$$
\mathcal{X}\left\langle S, S^{\prime}\right\rangle=\left\langle\widehat{\nabla}_{\mathcal{X}} S, S^{\prime}\right\rangle+\left\langle S, \widehat{\nabla}_{\mathcal{X}} S^{\prime}\right\rangle
$$

for any $S, S^{\prime} \in \mathfrak{A}$ and real $\mathcal{X} \in \operatorname{Der}(\mathfrak{A})$. This compatibility condition is equivalent to

$$
\omega(\mathcal{X})^{*}+\omega(\mathcal{X})=0
$$

for any real $\mathcal{X} \in \operatorname{Der}(\mathfrak{A})$. 
Any unitary element $U \in \mathfrak{A}$ with $\operatorname{det}(U)=\mathbb{1}$ defines on $\mathfrak{A}$ a right module endomorphism which preserves the hermitian structure and the det application by setting $S \mapsto U S$. We denote by $S U(\mathfrak{A})$ the group of such elements of $\mathfrak{A}$. This is exactly the gauge group of the $S U(n)$-vector bundle $E$. We denote by $U(\mathfrak{A})$ the group of unitary elements of $\mathfrak{A}$. For any $U \in U(\mathfrak{A})$, the gauge transformation of a noncommutative connection $\hat{\nabla}$ is defined by $\widehat{\nabla}_{\mathcal{X}}^{U} S=U^{*} \widehat{\nabla}_{\mathcal{X}}(U S)$. The noncommutative 1 -form $\omega$ is then transformed as

$$
\omega \mapsto U^{*} \omega U+U^{*} \hat{d} U
$$

The next proposition says that any (commutative) connection on $E$ defines canonically a noncommutative connection on $\mathfrak{A}$.

Proposition 9 For a fixed choice of a $S U(n)$-connection $\nabla^{E}$ on $E$, then of $\alpha$ as in Proposition 3, one defines a noncommutative connection $\widehat{\nabla}^{\alpha}$ by setting

$$
\widehat{\nabla}_{\mathcal{X}}^{\alpha} S=\nabla_{X} S+S \alpha(\mathcal{X})=\mathcal{X} S+\alpha(\mathcal{X}) S
$$

for any $\mathcal{X} \in \operatorname{Der}(\mathfrak{A})$ and $S \in \mathfrak{A}$.

The curvature of this connection is $\hat{R}^{\alpha}(\mathcal{X}, \mathcal{Y})=R^{E}(X, Y)$.

This noncommutative connection $\widehat{\nabla}^{\alpha}$ is compatible with the hermitian structure on $\mathfrak{A}$.

A gauge transformation on $\nabla^{E}$ induces a $S U(\mathfrak{A})$-gauge transformation on $\widehat{\nabla}^{\alpha}$.

Proof: This are just computations using the properties of $\alpha$ and $\nabla$.

These noncommutative connections are then other particular points in the space of noncommutative connections. Any other noncommutative connection $\hat{\nabla}$ can be decomposed as

$$
\widehat{\nabla}_{\mathcal{X}} S=\widehat{\nabla}_{\mathcal{X}}^{\alpha} S+\mathcal{A}(\mathcal{X}) S
$$

with $\mathcal{A} \in \Omega_{\text {Der }}^{1}(\mathfrak{A})$. In this case, one has

$$
\omega(\mathcal{X})=\alpha(\mathcal{X})+\mathcal{A}(\mathcal{X})
$$

Using (2), the supplementary term $\mathcal{A}$ can be decomposed itself as

$$
\mathcal{A}(\mathcal{X})=a(X)-B(\alpha(\mathcal{X}))
$$


Then $B$ is an application

$$
B: \mathfrak{A}_{0} \simeq \operatorname{Int}(\mathfrak{A}) \rightarrow \mathfrak{A}
$$

defined by $B(\gamma)=\mathcal{A}\left(a d_{\gamma}\right)$ for any $\gamma \in \mathfrak{A}_{0}$.

Physically, the $B$ term has been studied in [6] for the particular algebra $C^{\infty}(M) \otimes M_{n}(\mathbb{C})$. It was claim there that it can be interpreted as Higgs fields. In the present approach, one can see that $B$ is independent of the choice of the connection $\nabla^{E}$ on $E$, because it can be written $B(\gamma)=\omega\left(a d_{\gamma}\right)+\gamma$. The field $B$ is then a well defined object associated to $\widehat{\nabla}$ and corresponds to the "purely noncommutative" part of the connection in the trivial situation $C^{\infty}(M) \otimes M_{n}(\mathbb{C})$. In the general case, it is impossible to canonically split the connection into a "commutative" part and a "purely noncommutative" one. One has to choose an ordinary connection on $E$ to decompose it.

In a gauge transformation, one wishes to fix the connection of reference $\alpha$. In this case, the transformations relations of $\mathcal{A}, a$ and $B$ are given by

$$
\begin{aligned}
\mathcal{A} & \mapsto U^{*} \mathcal{A} U+U^{*}(\nabla U) \\
a & \mapsto U^{*} a U+U^{*}(\nabla U) \\
B & \mapsto U^{*} B U
\end{aligned}
$$

which are almost the ordinary gauge transformations of the connection 1-form for $\mathcal{A}$ and $a$, but with the ordinary differential replaced by $\nabla$.

In terms of $\alpha$ and $\mathcal{A}$, the curvature $\hat{R}$ of $\hat{\nabla}$ is given by

$$
\begin{aligned}
\hat{R}(\mathcal{X}, \mathcal{Y})= & \hat{R}^{\alpha}(\mathcal{X}, \mathcal{Y}) \\
& +\nabla_{X} \mathcal{A}(\mathcal{Y})-\nabla_{Y} \mathcal{A}(\mathcal{X})-\mathcal{A}([\mathcal{X}, \mathcal{Y}])+[\mathcal{A}(\mathcal{X}), \mathcal{A}(\mathcal{Y})] \\
= & R^{E}(X, Y) \\
& +(\hat{d} \mathcal{A})(\mathcal{X}, \mathcal{Y})+[\mathcal{A}(\mathcal{X}), \mathcal{A}(\mathcal{Y})] \\
& +[\alpha(\mathcal{X}), \mathcal{A}(\mathcal{Y})]-[\alpha(\mathcal{Y}), \mathcal{A}(\mathcal{X})]
\end{aligned}
$$


With the previous decomposition, one then has

$$
\begin{aligned}
\hat{R}(\mathcal{X}, \mathcal{Y})= & R^{E}(X, Y) \\
& +\nabla_{X} a(Y)-\nabla_{Y} a(X)-a([X, Y])+[a(X), a(Y)] \\
& -\nabla_{X} B(\alpha(\mathcal{Y}))-[a(X), B(\alpha(\mathcal{Y}))] \\
& +\nabla_{Y} B(\alpha(\mathcal{X}))+[a(Y), B(\alpha(\mathcal{X}))] \\
& +[B(\alpha(\mathcal{X})), B(\alpha(\mathcal{Y}))]+B(\alpha([\mathcal{X}, \mathcal{Y}]))
\end{aligned}
$$

Notice that the term $R^{E}(X, Y)+\nabla_{X} a(Y)-\nabla_{Y} a(X)-a([X, Y])+[a(X), a(Y)]$ can be interpreted as the curvature of the connection $\nabla_{X}^{\prime E}=\nabla_{X}^{E}+a(X)$ on $E$. Then the third and the fourth lines are expressions of the type $\nabla^{\prime}{ }_{X} B(\alpha(\mathcal{Y}))$.

In [6], for the trivial case $C^{\infty}(M) \otimes M_{n}(\mathbb{C})$, it was shown that the minima of the action written with this curvature is related to its horizontality. Then in the general case, let us look at the horizontality of the curvature $\hat{R}$. Taking $\mathcal{X}=a d_{\gamma}$ and $\mathcal{Y}=a d_{\eta}$ one gets the condition

$$
[B(\gamma), B(\eta)]-B([\gamma, \eta])=0
$$

and taking any $\mathcal{Y}$ one gets, using the previous relation,

$$
\nabla_{Y}^{\prime} B(\gamma)-B\left(\nabla_{Y} \gamma\right)=0
$$

which can be written

$$
\nabla_{Y} B+[a(Y), B]=0
$$

In case these two relations are satisfied, $\hat{R}$ is horizontal and

$$
\hat{R}(\mathcal{X}, \mathcal{Y})=R^{\prime E}(X, Y)-B\left(R^{E}(X, Y)\right)
$$

The relations (7) and (8) are the generalizations of the expressions found in [6] for the trivial case.

Notice that this computation has been done using the decomposition of the noncommutative 1-form $\mathcal{A}$ in $\omega=\alpha+\mathcal{A}$. It is possible to decompose directly the noncommutative 1-form $\omega$ as $\omega(\mathcal{X})=a_{\omega}(X)-B_{\omega}(\alpha(\mathcal{X}))$ and then compute 
the curvature. But the expressions of the horizontality conditions on $a_{\omega}$ and $B_{\omega}$ found in this way are less suggestive than (7) and (8).

One way to naturally generalize the previous discussion is to consider a right module of the type $\mathfrak{A}^{m}$ for $m \in \mathbb{N}$. This leads to similar results with much more structures, in particular in the Higgs sector (see [6] for the trivial case).

\subsection{The left module $\Gamma(E)$}

As a second canonical example, consider the space $\Gamma(E)$ of sections of $E$ which is a natural left $\mathfrak{A}$-module. Then one has the following result:

Proposition 10 There is a one-to-one correspondence between splittings of (5) as $C^{\infty}(M)$-modules and noncommutative left-connections on $\Gamma(E)$. The curvature of a noncommutative left-connections on $\Gamma(E)$ corresponds to the obstruction to be a Lie algebra splitting.

Proof: If $\widetilde{\nabla}$ is a noncommutative left-connection on $\Gamma(E)$, then $\widetilde{\nabla}_{\mathcal{X}}$ is a first order operator on $E$. Using the relation $\widetilde{\nabla}_{\mathcal{X}}(S e)=(\mathcal{X} S) e+S \widetilde{\nabla}_{\mathcal{X}} e$ for any $e \in \Gamma(E)$ and $S \in \mathfrak{A}$, one can show that $\left[\widetilde{\nabla}_{\mathcal{X}}, S\right]=\mathcal{X} S$. So $\mathcal{X} \mapsto \widetilde{\nabla}_{\mathcal{X}}$ is a splitting of (5) as $C^{\infty}(M)$-modules. If $D: \operatorname{Der}(\mathfrak{A}) \rightarrow \mathcal{A}(E)$ is a splitting of $(5)$ as $C^{\infty}(M)$-modules then $D(\mathcal{X})(S e)=[D(\mathcal{X}), S] e+S D(\mathcal{X}) e$ for any $S \in \mathfrak{A}$ and $e \in \Gamma(E)$. The first term is $(\mathcal{X} S) e$, and so $D$ satisfies the derivation rule of a noncommutative left-connection on $\Gamma(E)$.

Then the canonical splitting $\stackrel{\circ}{D}$ defined in Section 3 gives us a canonical noncommutative connection of vanishing curvature

$$
\stackrel{o}{D}(\mathcal{X}) e=\nabla_{X}^{E} e-\alpha(\mathcal{X}) e
$$

for any $e \in \Gamma(E)$ and $\mathcal{X} \in \operatorname{Der}(\mathfrak{A})$.

In this case, it is also possible to consider generalizations taking as left module a direct sum of $\Gamma(E)$. 


\section{Conclusions}

Here we have analyzed the noncommutative differential geometry of the algebra $\mathfrak{A}$ of sections of the endomorphism bundle of a $S U(n)$-vector bundle, thereby generalizing several results on the algebra of matrix valued functions. One advantage of doing this is to isolate what is canonical and what depends on the choice of a connection. It is worth noticing here that not every bundle in matrix algebras can be identified as the endomorphism bundle of a vector bundle (there are well known homological obstructions of that).

We have shown that connections on $S U(n)$-vector bundles identify with the affine subspace of $\Omega_{\text {Der }}(\mathfrak{A})$ of traceless antihermitian elements satisfying $\alpha\left(a d_{\gamma}\right)=$ $-\gamma$. This last condition is the analog of the vertical projection property of the corresponding connection form on the appropriate principal $S U(n)$-bundle. Notice however that here no equivariant property is required. In fact, it is apparent here that the noncommutative algebra $\mathfrak{A}$ can be used in many respects like this principal bundle. Doing that, we also make a bridge between the notion of Lie algebroids and the noncommutative differential calculus based on derivations. Furthermore, we have shown that the noncommutative connections on right or left modules are natural extensions of the usual connections.

Here we have restricted attention to the structure group $S U(n)$. But it is clear that by forgetting hermitian properties, one can pass to $S L(n, \mathbb{C})$, and by imposing reality, one can pass to $S O(n)$. 


\section{References}

[1] H. Cartan: in Colloque de Topologie, Bruxelles, 1950, Paris, Masson, p. 1951.

[2] A. Connes: Non-commutative differential geometry, Pub. IHES 62, 1986, p. 257.

[3] M. Dubois-Violette: Dérivations et calcul différentiel non commutatif, C.R. Acad. Sci. Paris, t. 307, Série I, p. 403-408, 1988.

[4] M. Dubois-Violette: Non-commutative differential geometry, quantum mechanics and gauge theory, in Differential Geometric Methods in Theoretical Physics, Proceedings Rapallo, 1990 (C. Bartocci, U. Bruzzo, R. Cianci, eds.), Lecture Notes in Physics 375, Springer Verlag, 1991.

[5] M. Dubois-Violette, R, Kerner, J. Madore: Noncommutative Differential Geometry of Matrix Algebras, J. Math. Phys. 31, p. 316, 1990.

[6] M. Dubois-Violette, R, Kerner, J. Madore: Noncommutative differential geometry and new models of gauge theory, J. Math. Phys. 31, p. 323, 1990.

[7] M. Dubois-Violette, P.W. Michor: Dérivations et calcul différentiel non commutatif II, C.R. Acad. Sci. Paris, t. 319, Série I, p. 927-931, 1994.

[8] K. MaCKEnZIE: Lie groupoids and Lie algebroids in differential geometry, London Mathematical Society Lecture Note Series, 124, Cambridge University Press, 1987.

[9] T. Masson: Submanifolds and Quotient Manifolds in Noncommutative Geometry, J. Math. Phys. 37, 5, p. 2484, 1996. 